\title{
Evaluating measurement models in animal personality: operational, latent variable and network approaches
}

\author{
Conor Goold \\ Faculty of Biological Sciences, University of Leeds, Leeds, LS2 9JT, United Kingdom
}

\begin{abstract}
Animal personality is the study of consistent individual differences in behaviour. Underlying this innocuous definition, however, is a history of diverse scientific ideas and terminological confusion that continues to elicit debate about the usefulness of personality in animal behaviour. This review connects these debates to the absence of a unifying measurement model for animal personality. A measurement model is a blueprint for how theoretical constructs and observed variables relate to each other, and thus makes the assumptions of scientific theories explicit. The field of animal personality is demonstrated to employ two predominant measurement models, operational and latent variable. Each framework harbours its own conceptual problems that are shown to contribute to debates about animal personality. Five requirements for a suitable measurement model for animal personality are subsequently provided. The review ends by considering the utility of the network approach arising in human psychology as an alternative measurement framework for animal personality.
\end{abstract}

\section{Introduction}

Over the last two decades, individual differences in behaviour has become a dominant research paradigm in animal behaviour. Widely referred to now as animal personality, evidence that individuals differ relatively consistently in their behaviours from others is of interest to a range of scientists in evolutionary and and behavioural ecology, animal cognition, behavioural physiology, and applied animal welfare science. Beyond recording consistency in individual behaviours within populations (e.g. risk-taking [1]), animal personality research is concerned with the non-trivial questions of whether behavioural consistency is an evolutionary adaptation or constraint [2, 3, 4], the relative importance of genetic compared to situational factors in maintaining behavioural consistency [5], why organismal behaviour appears globally organised into correlated 
suites of traits (i.e. behavioural syndromes; [6]), and why similar behavioural dimensions are found in diverse taxa [7, 8].

The rise of animal personality has not been without contention, however, with a number of papers attempting to clarify the personality concept [9, 10, 11, 12, 13], particularly in its distinction from human personality psychology, or even questioning the value of animal personality as a science [14, 15]. Proponents of personality highlight the importance of behavioural consistency, more precisely behavioural repeatability (the fraction of total behavioural variance attributable to between-individual differences; [16]), in its own right and how it relates to the heritability of phenotypic traits of interest in quantitative genetics [5, 11]. The criticisms levelled against personality have included claims that is it overly anthropomorphic [15], with terminology serving only to increase media accessibility [14], or that the field brings no new insights about animal behaviour beyond what can be inferred by more established disciplines [14, 17, 15]. Others have identified a lack of conceptual cohesivenesss between personality studies [12], or addressed the need for more hypothesis-driven frameworks for understanding behavioural repeatability [18, 19]. While rebuttals to these criticisms have rightly indicated that personality studies have spawned a new form of individual-oriented research in fields like behavioural ecology [20, 21], it is clear that across animal behaviour, personality remains a controversial topic.

The goal of the present review is not to contribute to the confusion about animal personality, but instead to highlight a broader cause of this dissension: that animal personality science has no unified measurement model. A measurement model is a theoretical device that links unobserved variables to observed variables [22, 23, 24], and provides a blueprint for scientific theories [22]. Human psychologists devote an entire area to the question of psychological measurement - psychometrics — but what it means to measure something in animal behaviour has not received the same level of focus. Regardless of which side researchers align with on the contention about animal personality, there has been a consistent call for more theoreticallyjustified and hypothesis-driven personality research focused on the mechanisms and adaptive significance of repeatable individual differences [11, 25, 26, 27, 28, 19, 29, 14, 12, 30, 31, 32]. It is argued here that defining a clearer measurement model for animal personality, specifically the relationship between personality traits, observed behaviours, and behavioural repeatability, will greatly enable this endeavour.

Section 2 discusses the dominant definition of animal personality and personality traits from behavioural and evolutionary ecology as any observable behaviour that is repeatable [11, which aligns with an operationalist measurement model. After raising well-known conceptual problems that operationalism induces, section 3 reviews the latent variable approach to personality. Latent variable modelling has been used in human psychology for over a century, and thus the field has learned of the problems with this form of measurement model, both in theory and in practice, that are infrequently discussed in animal personality. Lastly, the final section examines what an ideal measurement model in animal personality needs to provide, and investigates the 
utility of an alternative network approach that has risen within human psychology over the last decade [33, 34, 35, 36, and has recently been used in animal behaviour [37, 38]. It is important to note that this review does not intend to equate animal personality with human psychology, nor does it ignore the unique history animal personality science has in its own right [39, 21]. Instead, it illustrates the common conceptual problems faced by both fields, irregardless of their differences, as empirical sciences grappling with the difficult task of measurement, and motivates a deeper appreciation of measurement in animal personality to provide a more constructive foundation for discussing theoretical issues in the future.

\section{The operational approach}

Individual variation in behaviour is a core tenet of evolutionary biology, but the proposal that individual variation may be adaptive has only gained widespread traction in the last two decades $[1,40,6,41,3]$. This is not to imply that individual variation in behaviour was not of interest before this time, however. Psychologists, ethologists, stress physiologists, applied animal behaviourists and others had in fact been writing on the presence of individual variation in behaviour and 'personality' for decades [42, 43, 44, 45, 46, 47, 48, 49, 50, 51, 52. In large part, these studies were a direct analogue to studies of human personality because the goal was to examine whether animals showed similar personality trait structures to humans [52]. The hypothesis that individual variation may be adaptive, rather than just noise around an adaptive peak, was also discussed in evolutionary biology 40 years ago [53, 43]. This was a timely extension in using "natural selection to explain differences between organisms at increasingly finer scales" [40, p. 199].

The introduction of animal personality into studies of behavioural and evolutionary ecology at the scale seen today was greatly facilitated by Reale et al. [11]'s seminal article, which operationalised personality (referred to as 'temperament' in the article) in terms of purely observable behaviours. Whereas psychologists referred to personality traits as latent psychological properties of individuals, Reale et al. [11, p. 294] defined animal temperament as "the phenomenon that individual behavioural differences are consistent over time/across situations", and proposed five initial traits that most behaviours reflect (aggression, sociality, boldness, exploration and activity). Rather than interpret these traits as " 'unobservables' or qualities that are difficult to measure (e.g. dispositions)...[that] may restrain ecologists and evolutionary biologists from studying temperament" [11, p. 294], the authors emphasised that "each trait should be operationally defined and its ecological validity tested" (295). Importantly, this definition of personality is a population-level phenomenon, and not something that exists at the level of individuals.

The distancing of animal from human personality was reinforced by subsequent authors [9, 10, 20]. For instance, Carter et al. [10, p. 468] wrote that the term 'trait' "is used in behavioural ecology to mean a measurable aspect of an individual's be- 
haviour that is, usually, repeatable while in psychology the use is more abstract and describes a construct". Bell [20, p. 626] further emphasised that behavioural ecologists do not infer any higher-order cognitive processes underlying personality: "We are agnostic about the sources of variation... and how long the differences endure". Operationalising animal personality as any measurable behaviour that is repeatable allowed integration to established concepts and statistical formulations in quantitative genetics. Notably, the variance of between-individual differences in behaviour is equal to the sum of additive, permanent environmental and genetic dominance variance [5]. From the definition of heritability as the ratio of total genetic variance to phenotypic variance (broad sense) or the ratio of additive genetic variance to phenotypic variance (narrow sense), behavioural repeatability was defined as the ratio of between-individual variance to total phenotypic/behavioural variance. Repeatability is readily calculated from the variance components of hierarchical statistical models 16, 54.

The operational approach has no doubt been a powerful empirical paradigm for quantifying individual variation in behaviour. Its use of hierarchical statistical modelling has also integrated personality with the concepts of behavioural plasticity [55] and predictability [56, 57], continuing the expansion of understanding how natural selection maintains behavioural variation across multiple hierarchical levels 40, 58, 59, 29, 60, 61. This has been concomitant with a shift in behavioural ecology study design to collecting repeated measures of often multiple different behaviours 21]. Nonetheless, personality in behavioural and evolutionary ecology has received criticism, including that the term personality continues to invoke anthropomorphic interpretations of behaviour [15], and that studies quantifying behavioural repeatability are overly descriptive [25, 14, 18], resulting in statistical estimates of behavioural repeatability with little explanatory value.

For instance, there are few guidelines about what constitutes sufficient behavioural repeatability to be evidence of personality. Variance components, and thus repeatability, can never be exactly zero, making finding a suitable null hypothesis difficult [14]. There is also a lack of theoretical rationale for why different values of repeatability are found [18]. Despite some theoretical models explaining when individual differences should evolve [26], those models do not make numerical predictions about the repeatability coefficients in particular. While repeatability is often around 0.350.4 [62], a priori numerical predictions of repeatability beyond this moderate value are rare to come by. Indeed, because repeatability frequently accounts for less that $50 \%$ of behavioural variance, there are questions of how much emphasis should be placed on repeatability for predicting behaviour [63]. Quantifying repeatability, and labelling repeatable behaviours with terms such as boldness or exploration, has thus motivated some to question the worth of personality at all [14].

The aforementioned criticisms could rightly be made about many different fields of empirical research, and some valid responses to these criticisms have been provided [21, 39, 20]. However, a broader reason driving such criticisms becomes evident when 
one assesses the type of measurement model the operational approach defines: the operational approach is a re-emergence of operationalism, the now-rejected philosophy of science originating in the 1920s with Bridgman's 1927 book The Logic of Modern Physics. Bridgman proposed that theoretical constructs are synonymous with the methods used to measure them:

To find the length of an object we have to perform certain physical operations. The concept of length is therefore fixed when the operations by which length is measured are fixed: that is, the concept of length involves as much as and nothing more than the set of operations by which length is measured.

- Bridgman [64, p. 5]

At a time when psychological testing (e.g. to predict educational attainment) was rampant under the logical positivist and verificationist movements, human psychologists promoted operationalism because it allowed the "illusion of control over the perplexities of the "real world" " 65, p. 150] by reducing theoretical ideas (e.g. intelligence) to observables (e.g. scores on intelligence tests). As Stevens [66, p. 323] wrote, this "ensures us against hazy, ambiguous and contradictory notions and provides the rigor of definition which silences useless controversy".

Early commentators on the rise of operationalism in physics [67] and psychology [68] explicated its problems, and philosophers and psychologists have continued to publish on its problems as a philosophy of science throughout the decades [69, 70, 71, 22, 72]. The 'tragedy of operationalism' [73] is that it equates meaning with measurement [69], rendering scientific constructs ontologically ambiguous [22]. To say that the concept of length is defined by the methods used to measure it automatically means that every different method used to measure the length of an object, including two different people or the same person at two different times, necessarily results in a different length concept [69, 22, 23]. Moreover, operationalism revokes the idea that one measurement can be 'better' or 'worse' than another, because both measurements are equally synonymous with the construct under study by definition [69]. Concepts such as validity and reliability thus fall by the wayside under operationalism [23]. In terms of measurement models, operationalism provides no such thing [74]: equating the theoretical construct with the observed measurements absolves the need for the construct entirely.

In practice, most scientists would find the operationalist doctrine, taken to its logical conclusion, nonsensical. Indeed, the logical positivists removed support for operationalism once its problems were revealed, and even Bridgman relaxed his ideas in later years [69, 71]. Scientific constructs cannot be exhausted by their operational definitions 72 because at some point the concept has to be distinguished from the methods or operations employing it [69, 73]. Borsboom [22] has discussed the problems operationalism has incited in psychology's classical test theory, and in the common practice of operationalising theoretical attributes and using the measures 


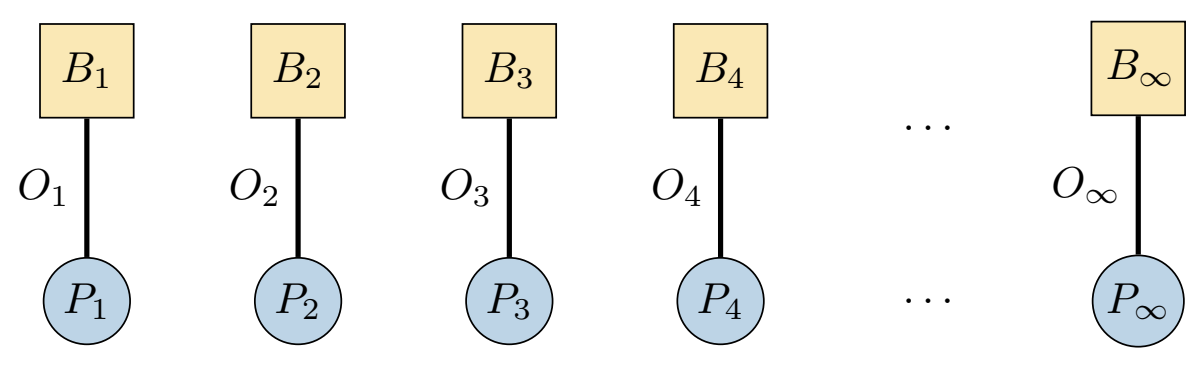

Figure 1: A schematic representation of the 'trajedy of operationalism'. Imagine a single personality trait, $P$, which is operationally defined (solid lines; $O_{i}$, from $i=1, \ldots, \infty$ ) by different observed behaviours $\left(\mathbf{B}=\left\{B_{i}\right\}\right.$, from $\left.i=1, \ldots, \infty\right)$. Operational definitions synonymise the personality trait with each observed behaviour, precluding understanding the causal relationship between $P$ and measures $B_{i}$ (e.g. $P_{i} \rightarrow B_{i}$ or $P_{i} \leftarrow B_{i}$ ) or allowing inference of how a single personality trait may influence all behaviours (e.g. $P \rightarrow \mathbf{B}$ ). Due to an infinite number of ways of measuring behaviour, there are also an infinite number of concepts (indicated by $P_{\infty}$ ) instantiated operationalism.

as synonymous with those attributes [74]. Outside psychology, Stafleu et al. [75] remark that operational approaches have eroded the concept of animal welfare of its ethical aspects. There is, furthermore, close relationships between common methods of assessing validity, such as construct validity, and operationalism, which have also been highly criticised [76, 23].

Defining personality directly in terms of behavioural repeatability is another instantiation of operationalism. Indeed, Reale et al. [11]'s support for operationally defining traits to avoid eliciting notions of psychological dispositions shares significant similarities with the reasoning for adopting operationalism in psychology discussed above. It is crucial to recognise that this is not a claim that behavioural repeatability is a-theoretical, or that its importance to understanding patterns of behavioural variation in behavioural and evolutionary ecology (e.g. [59, 60, 58]) should be ignored. It is a claim that making behavioural repeatability sufficient, and not simply necessary, to define animal personality is another demonstration of operationalism. This idea is shown schematically in graph-theoretic form in Figure 1. Here, an infinite number of behaviours are bound (thick black lines) to separate personality traits, with no way of determining the relationship between traits nor the causal relationship between traits and behaviours (i.e. it is intentionally not a directed graph).

210 Arguments that personality in behavioural and evolutionary ecology has resulted in a literature that is too descriptive, or that there is a need for more hypothesis-driven approaches [18, 19], is a function, I argue, of the more general problem of operationalism: if repeatability is sufficient to define a personality or trait, then nothing more is required of researchers than to estimate repeatability. That the field of personality has too many descriptive studies, with apparently meaningless trait vocabularies 
tacked on [14], is no surprise if the dominant measurement concept is operationalism. Assessing validity and reliability cannot ameliorate these matters, as has been argued previously [10], because operationalism obfuscates any notion of more than one measure of the same construct, or even of two different raters measuring the same construct [22, 76]. By extension, operationalism distorts our understanding of behavioural correlations, or behavioural syndromes, because every correlation between two repeatable behaviours must in fact represent an among-individual correlation between two different traits or a syndrome. There are an infinite number of ways we can measure behaviour, and therefore there must be an infinite number of behavioural syndromes if operationalism is to be adhered to.

Wanting to link repeatable behaviours to higher-order behavioural dimensions, on the one hand, and following an operationalist approach, on the other, causes conceptual tension that is seen in empirical research. One example is highlighted by Dochtermann and Nelson [77, who found that two measures of exploration were repeatable but, unexpectedly, uncorrelated. The authors comment that the lack of predicted correlation "makes determining whether 'exploratory behaviour' is generally a trait problematic" [77, p. 1115], and they suggest that more attention should be placed on understanding what traits are, as they have been in human personality psychology. In this instance, the operational definition has eliminated any inferen235 tial power to make sense of the lack of clear correlation between behaviours. One cannot conclude that exploration is not a trait, because both behaviours meet the requirement for exploration under the definition of behavioural repeatability, and are ecologically valid. Yet, at the same time, the evidence does not support that exploration is a trait because the absence of a significant correlation between two ecologically connected behaviours contradicts the pragmatic notion that traits can be measured by multiple behaviours. To enable a clearer conclusion would require an explicit measurement model of personality that distinguishes between exploration, as an unobserved variable quite separate to scientific practice, and the measures of exploration, as indeed the authors indicate later in their article.

245 Operationalism provides no clear measurement model for personality science, and I have argued that this has been a driving force in fueling debate between personality researchers. Importantly, this argument is agnostic to the fact that behavioural repeatability and animal personality is motivated from evolutionary biology, whereas human personality is motivated by notions of psychological dispositions [11]. Therefore, claims that criticisms of animal personality misleadingly ignore these disciplinary differences [39] do not apply. The remedy to operationalism is to align with a philosophy of science and measurement approach that allows distinguishing the concept from measurements of that concept. 


\section{The latent variable approach}

Animal personality researchers already have a more suitable measurement model than operationalism in their toolkits. Alongside calculating behavioural repeatabilities using hierarchical statistical modelling, another approach to studying personality involves collecting multivariate data on behaviour and explicitly decomposing the variance into underlying behavioural dimensions [78]. Note that this approach is immediately at odds with an operationalist definition by introduction of unobserved variables into the measurement process. This latent variable modelling offers a principled statistical methodology for relating unobserved or latent variables to observed or manifest variables [79]. Beyond its data analysis applications, latent variable models also provide a principled method of thinking about the measurement relationships between variables, such that theoretical relationships can be tested [22]. Latent variable models have, consequently, been a mainstay of human psychology for over a century [80, 81, including personality psychology, for inferring latent psychological constructs from observed measurements, such as the infamous Five Factor Model of human personality [82. Moreover, early applications of personality to animal behaviour by comparative psychologists, ethologists and applied animal behaviourists used latent variable models [47, 42, 49].

In recent years, latent variable modelling has also increased in popularity in behavioural ecology to characterise personality traits and behavioural syndromes [7, 83, 84, 29, 85, 32. Therefore, despite differences between human psychology and animal behaviour in studying personality, there appears to be a convergence in methodology to using latent variable modelling [29]. However, whereas latent constructs in human personality have been expressed as real, biological variables [82], there has been a stark lack of discussion in animal personality over what latent traits derived from latent variable models refer to in animal behaviour, or whether latent variables are causal variables or composite variables with no causal status (although see [29]). As is known from human psychology, both these problems cause confusion about what personality and personality traits refer to. For example, latent variable modelling results in processes of 'verbal magic' [86] when naming latent factors, and unclear causal relationships between latent and manifest variables results in model misuse [74].

Latent variable models generally come in two different flavours depending on the causal relationships they indicate between latent and manifest variables, as shown by the directed acyclic graphs in Figure 2. Formative models, most commonly employed as principal components analysis, assume that the latent variables are caused by changes in the manifest variables (Figure 2a). Reflective models, most commonly employed as factor analysis for a continuous latent variable, assume that variation in the manifest variables are caused by changes in the latent variable (Figure $2 \mathrm{~b}$ ). There are no assumptions that manifest variables should be correlated with one another in formative models [79], but manifest variables should be conditionally indepen- 


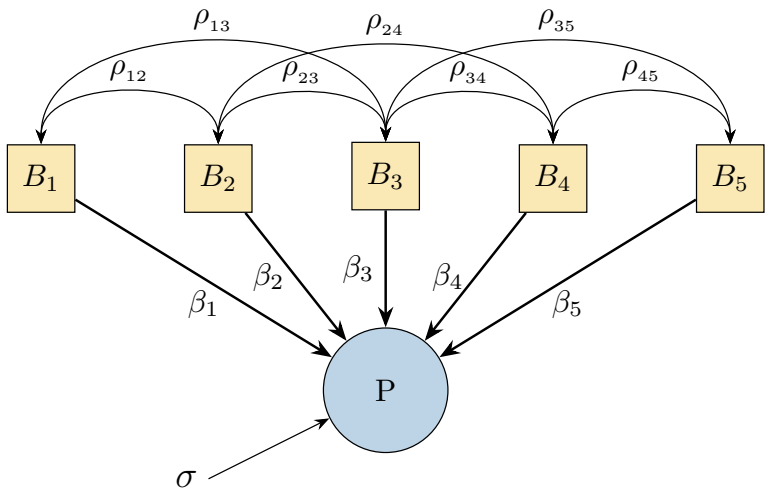

(a)

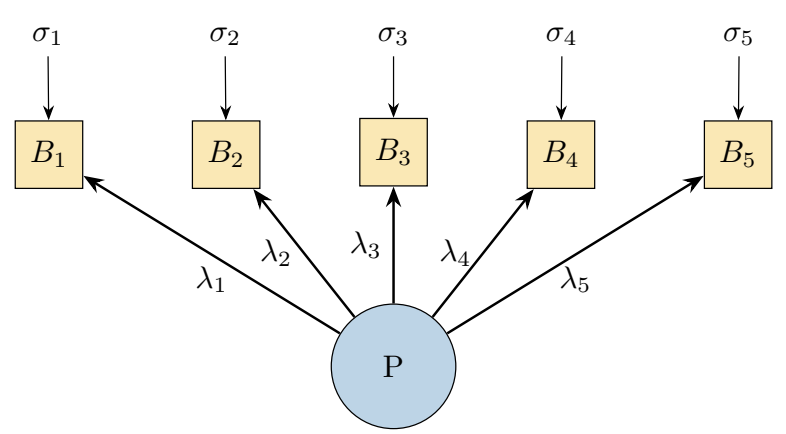

(b)

Figure 2: Formative and reflective latent variable models, adapted from Bollen \& Lennox 79. Formative models assume the latent personality trait is composed of a linear combination of perfectly-measured manifest behavioural variables (i.e. $\sum_{i}^{P} \beta_{i} B_{i}$ ). The manifest variables represent only a subset of (possibly covarying, i.e. $\rho_{i j}, i \neq j$ ) variables that could compose the latent trait, so the latent trait is measured with error $(\sigma)$. Reflective models assume that variation in the manifest variables are caused by changes in the latent personality trait (i.e. $\sum_{i}^{P} \lambda_{i} x$, where $x$ is the latent variable). The manifest variables are not completely described by variation in the personality trait, and so can be measured with error $\left(\epsilon_{i}\right)$, and are assumed conditionally independent given the latent variable.

dent given the latent variable(s) in reflective models. Authors in human psychology [87, 79, 88, 89, 74] and some now in animal behaviour [78, 84, 90] (see also [91]) have recognised the continued conflation of these opposing models, not helped by inconsistent language in popular statistical analysis software [74]. It is not just potential differences in the statistical results that are important (e.g. [89, 90]; but see [92, 93]), but that the causal status of the latent variables are frequently ignored [94]. This necessarily increases confusion over what latent variables represent when discussing animal personality.

For the most part, psychologists agree that psychological constructs, like personality traits, are best viewed as reflective latent variables [22, 94, 86, 87, 95]. Such a view necessitates a realist interpretation of traits - that is, traits are real biological variables distinct from the methods and procedures of scientific practice [22, 72, 86]. This purview is most powerful within a confirmatory paradigm, because the causal hypothesis of the latent variables allows us to test certain assumptions using reflective latent variable statistical models. For example, confirmatory factor analysis or, more generally, structural equation modelling can be used to test the assumptions of local independence [96] and measurement invariance [97, 98, 99, 100]. Local independence states that manifest variables are conditionally independent given the latent variable, meaning accounting for the latent variable in the directed acyclic graph of Figure 
$2 \mathrm{~b}$ should 'screen off' any correlations between the manifest variables. Formally, the probability, $\pi$, of two manifest variables, $B_{1,2}$, given a latent variable, should be factorisable as $\pi\left(B_{1}, B_{2} \mid P\right)=\pi\left(B_{1} \mid P\right) \pi\left(B_{2} \mid P\right)$. Measurement invariance is, by contrast, the assumption that any differences between groups (e.g. populations, individuals, experimental groups) are functions of differences in the latent variable rather than in other aspects of the measurement relationship. Formally, given some specific value of a latent variable, $P_{x}$, and two different groups, $G_{1,2}$, measurement invariance implies that $\pi\left(B \mid P_{x}\right)=\pi\left(B \mid P_{x}, G_{1,2}\right)$. If measurement invariance does not hold, then there must be other processes going on in the measurement process that affect the observed behaviours, and one cannot infer that group differences are functions solely of the latent variable [22]. Differences between majority and minority ethnicities on intelligence tests may not be a function of underlying intelligence, for example, but due to differences in reading comprehension related to language abilities [101].

The application of latent variable modelling in animal personality is less regulated and rigorous than its application in human psychology and psychometrics, despite calls for their better use [78, 90]. Although reflective models are used by a number of researchers [83, 84, 60, 85, there has been too little discussion about the a priori justification, or lack thereof, of choice of formative (particularly principal components analysis) and reflective models in animal personality [78]. Assessments of measurement invariance and local independence, despite a number of studies comparing traits across species, populations and contexts, are sorely lacking (but see Goold \& Newberry [102]).

One exception worth examining is that of Araya-Ajoy and Dingemanse [29], who provide a framework for investigating latent behavioural 'characters' using a two-step multivariate hierarchical regression and structural equation modelling approach. The authors clearly outline the assumptions a latent variable interpretation of behavioural characters imposes, such as that correlations between behaviours should be similar in different contexts and environments if in fact a latent variable is causing behaviour. Despite this, the authors provide no reference to the already long history of testing these assumptions in latent variable models in human psychology, namely local independence and measurement invariance. The authors further state that their proposed 8], particularly with respect to modelling between- and within-individual correlations. This is in fact false: human psychologists have proposed a variety of tools based on structural equation modelling that can estimate between- and within-individual variation in latent and/or observed variables across time and contexts, such as dynamic factor analysis [103] or state space models [104. These methods allow for different assumptions to be tested within the same statistical model, rather than the two-step approach presented by Araya-Ajoy and Dingemanse [29.

Latent variable modelling has, therefore, untapped potential to offer personality researchers in animal behaviour to test explicit theoretical hypotheses about how observed behaviours are caused by unobserved, latent traits or characters [102. As- 
certaining local independence and measurement invariance could offer new avenues for testing the comparative structure of personality across species (i.e. do the same measurement relationships hold in different species such that the same trait can be inferred across species). Validation of personality assessments can also make use of latent variable modelling in line with modern psychometric ideas [76, 94], rather than relying on inspecting patterns of inter-correlations between variables [10]. This is not an argument for more advanced statistical models, but motivation for making measurement relationships between unobserved traits and observed behaviours explicit.

\subsection{Problems with latent variables}

The latent variable approach provides a more principled measurement model for understanding personality and personality traits. It is worth addressing, however, some pressing concerns about latent variables that have been raised in human psychology. Namely, if latent variables are to be interpreted in a realist sense, where is the evidence supporting the idea of homogeneous, 'functional units' [29] in biology?

If latent variables are real, biological variables, they presumably must exist at the level of individuals. However, most studies applying latent variable models do so at the population level, rather than imposing a hierarchical structure where estimation of the latent variables varies across subjects. For example, Molenaar [105] demonstrated that the Five Factor Model of human personality, considered a 'universal structure' of psychology [82], does not hold for within-subject data [104]. This is a generalisation of the ecological fallacy [106, 107], and arises because behaviour is 'non-ergodic' [105, 108, meaning the average value across a set of units (e.g. animals) does not equal the average of repeated realisations of each individual unit. Although Araya-Ajoy and Dingemanse [29] proposed a hierarchical structure for behavioural variation that includes latent behavioural characters, the latent variables were still estimated at the population level. Thus, there have been no applications of the hierarchical latent variable models as proposed by Molenaar [105] in animal personality. Employment of such modelling schemes would more rigorously test whether latent behavioural traits or characters hold at the individual level.

If a within-individual interpretation of latent variables is permitted, latent variables still appear to be an inaccurate interpretation of the biological mechanisms underpinning behaviour. A notable example is general intelligence, also known as the $g$-factor. Despite over a hundred years of research on $g$, a homogeneous biological $g$ is yet to be found [109, 110, 111]. Brain networks underlying intelligence and other cognitive functions vary across people and contexts [112, 113], and are dynamicallydistributed across the brain [114]. This emerging evidence questions the functional modularity of the brain [115, 116] and suggests that $i$ ) the same biological structures have multiple different functions, known as degeneracy, and $i i$ ) different biological structures have similar functions, known as redundancy [117, 118, 119]. Representing complex mechanisms by the relatively fixed structures of latent variables is to com- 
mit the 'fallacy of misplaced concreteness' [120], a prominent source of confusion that has been identified as distorting empirical progress on concepts as wide-ranging as human intelligence [121, forest biomass [122], and economic equilibrium [123]. It is also to take out a 'loan on intelligence' [124, by equating unobserved processes with brain-based, cognitive processes without sufficient evidence.

Latent variable models will likely continue to be a dominant measurement model despite problems with the realist interpretations of latent variables. However, there are alternatives to latent variable perspectives. In the final section, I will outline the requirements for an ideal measurement model for animal personality based on the preceding discussion, and take a brief look at the rising network approach in human psychology that has been proposed as an alternative paradigm to latent variable models.

\section{Alternative measurement models for animal personality}

410 The discussion above relates more generally to the growing call for explicit theoretical models to drive hypotheses in empirical science. Theoretical models encode causal hypotheses of how complex systems function [125, 126, and encourage more reproducible science by facilitating practices such as pre-registration [126]. Golonka and Wilson [127] argue for a better 'guide to discovery' in human psychology based on clearer theoretical roadmaps. Theoretical models of how behavioural variation and, thus, animal personality evolves do exist [26, 4, 128, 129, 130]. However, there is a disparity between these models and the majority of personality studies that rely on quantifying behavioural repeatability alone, or fitting a latent variable model with few of the checks and balances required to validate a latent variable interpretation of behaviour [94. There is also a disparity between recognition of the complex genetic, physiological and neurobiological mechanisms underlying traits [11], and the types of measurement models employed.

\subsection{Measurement model requirements}

Five requirements for a measurement model in animal personality can be inferred

from the preceding sections. The measurement model must ideally:

1. Reference both the theoretical construct and the observed variables

2. Apply to individuals, and not aggregates (e.g. populations)

3. Encode a causal hypothesis about how observed behaviours are expressed

4. Be dynamic, based on time and/or context-varying influences 
5. Allow for the inclusion of how other variables, such as biological and environmental factors, influence focal behaviours

The operational approach excels at evaluating the time- and context-varying aspect of behaviour (requirement 4) by virtue of its use of hierarchical modelling, and such models can also include predictors of individual variation (requirement 5) as

\subsection{The network approach}

Multiple areas of scientific inquiry have converged upon a network view of complex systems, such as evolution, communication systems, physiological and neurobiological 


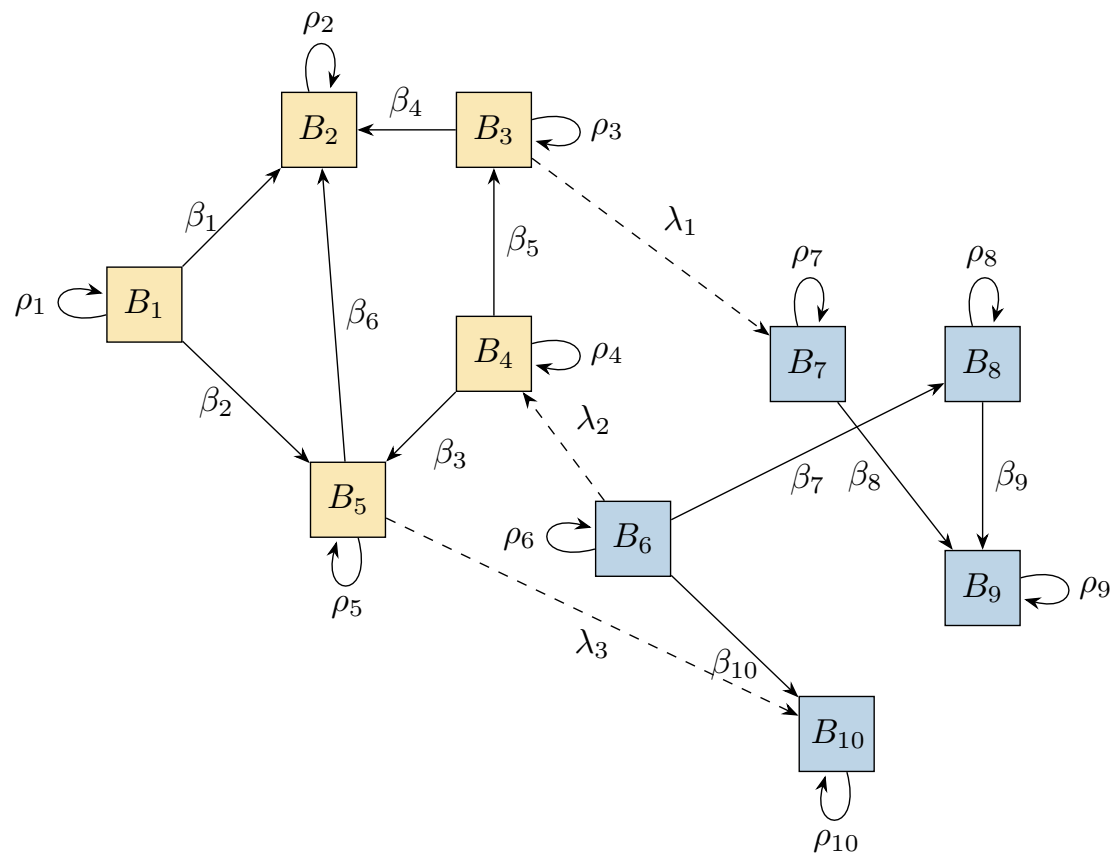

Figure 3: A hypothetical individual-level network of behavioural variables (squares labelled $B_{i}$ ) clustering into two emergent 'traits' (yellow and blue squares). Each behaviour has a within-behaviour effect through time/across contexts (self-directed loops denoted $\rho_{i}$ ), and between-behaviour causal effects (directed arrows denoted $\beta_{i}$ ). Dashed lines represent causal effects between traits.

systems, and developmental systems [132, 131, 133, 114, 134, 135, 136]. This is not only due to the utility of network statistical models, but reflects a realisation that systems are composed of multiple interacting entities that frequently show nonlinear and emergent behaviours [135]. Philosophers have also revived interest in process-based philosophies of science [120, 137, 138] that assert that the natural world, including animal behaviour [139], is not composed of fixed substances or 'things', but self-maintaining processes that re-emerge through time ("enduring things are never more than patterns of stability in a sea of process" [137, p. 14]). A network perspective is a natural representation of processes (e.g. microbiology; [140]).

Can behaviour also be thought of as a network? Over the last 10-15 years, and in opposition to latent variable interpretations of psychological constructs, a dynamical systems account of psychology based on networks has been formulated [110, 33, 34, 35, 36]. The network approach suggests that the behavioural, emotional and cognitive variables within individuals comprise a dynamic, self-organising web of interacting components. Specifically, each variable can be represented by a vertex or node, and edges connecting nodes represent real causal relationships (Figure 3). By 'causal', the network approach posits that the occurrence of one variable (e.g. feeling sad) 
can directly lead to the increased chance of another variable (e.g. sleeping less), conditional upon all other variables in the network. The network approach therefore rests on principles of causality based on conditional independence relationships on directed acyclic graphs instantiated by Pearl [141].

Functionally similar network components are predicted to hold stronger causal relationships, forming clusters of recurring components through space and time within individuals. Clinically depressed individuals, for instance, might show consistent feedbacks between symptoms, such as 'fatigue' $\rightarrow$ 'concentration problems' $\rightarrow$ 'low mood' [142]. Depression is not some latent variable to 'have' or 'not have', therefore, but may be an emergent property of habitually expressed symptoms through time and space [142, 143], in the same way that collective animal behaviours are emergent properties of relatively simple rules between individuals or functional brain networks are emergent properties of synaptic activity [117]. Cross-correlations between constructs, such as comorbidities between generalised anxiety and depression, which complicate assumptions of unidimensionality in reflective latent variable models (i.e. manifest variables should only load on one factor), are naturally handled in the network view as it does not constrain correlations between diverse components and recognises the fuzzy boundaries between many traits [34, 144]. Networks are additionally equipped to handle problems of degeneracy and redundancy [117, 118] because different components may contribute to the same component-clusters, and the same component can contribute to different clusters.

The measurement relationship in this instance is neither operational, formative nor reflective, but mereological: "the observables do not measure the construct, but are part of it" [145, p. 47] (see also Borsboom \& Cramer [146] or Borsboom et al. [36]). This is not operationalism disguised: computing a network of relationships between variables is not enough to evidence the network approach, and in fact network models can be shown to be statistically equivalent to some latent variable models [147, 148. Instead, the network approach asserts that causal relationships exist be515 tween behaviours through time and space, and it is the nature of these causal processes (e.g. exciting or inhibiting) that define the organisation of behaviour. This causal behavioural system exists independently of the operations used to characterise it. Borrowing from Boyd's [149] defense of viewing natural kinds (e.g. species) as 'homeostatic property clusters', Kendler [150, p. 1147] argued that psychiatric disorders were best understood as mechanistic property clusters: the properties (symptoms) "are not similar merely in their superficial properties...but because the co-occurrence of these properties from individual to individual is explained by causal mechanisms that regularly ensure these properties are instantiated together". The causal mechanisms dictating behaviour may indeed include genetic, neurobiological or physiological 525 variables as part of the causal behavioural system. However, these variables also exist in their own right and are not relegated to latent variables.

The network approach has burgeoned into a highly active discipline with widespread theoretical [151], fundamental [36, 152], and clinical [153, 144] significance to human 
psychology. Network models are employed using causal modelling techniques such as the Gaussian graphical model for metric variables, the Ising model for binary variables, and multivariate vector autoregressive models for time-varying data [151. Network models have, furthermore, been applied in a hierarchical structure [154], which demonstrated inter-individual variation in network structures (e.g. the sign and magnitude of partial correlations between behaviours). In terms of the requirements for a suitable measurement model for animal personality, networks models account for the theoretical construct and observed variables (the construct is an emergent property of the network), apply to individuals and not to aggregates, are causal hypotheses of behaviour, can be applied to time- or context-varying data, and also allow one arbitrary flexibility to include additional variables that influence behavioural variables. For example, Isvoranu et al. [155] include environmental risk factors into their network model of psychosis, such as developmental trauma. Although network models have faced their own criticisms [148, 147, 156], they have received widespread support from across human psychology, with researchers actively working on network theory, methodology and replicability [151, 157].

Few studies have considered the network approach to understand behavioural phenotypes in animals. Wilkins et al. [158] presented a network approach to understand multi-modal communication signalling in American barn swallows (Hirundo rustica erythrogaster), and others have encouraged a systems approach to animal communication [159]. Goold et al. [37] used network analysis to understand cross-sectional owner reports of working dog behaviour, including the use of network centrality measures and estimation of network robustness. More recently, Martin et al. [38] motivated a combined exploratory graph analysis - generalised network approach [151] that combined factor analysis with network models of residual pairwise correlations to investigate behavioural syndrome structure in common marmosets (Callithrix jacchus). The latter authors provide a cohesive overview of the utilities of network modelling in addition to latent variable modelling on statistical and empirical grounds.

Given the growing support for network models in human psychology, as well as across scientific areas, the network approach may offer a unifying measurement model to understand animal personality, behavioural syndromes, and individual variation. Hierarchical network models [154] would allow the continued estimation of individual-varying parameters to conduct variance components analyses and calculate behavioural repeatability. Correlations between behaviours, such as behavioural syndromes, are naturally represented because the core of network approaches is estimating regularised networks of partial correlations between behaviours. Importantly, the theoretical concept of state-behaviour feedback loops [130] should find immediate application in network models by including state variables directly as network variables. Lastly, network models are not completely opposed to latent variables, meaning latent variables may also be included where appropriate or practically useful (e.g. Martin et al. see [38]). While a lot of work remains to be done to formulate a network measurement model for animal personality, there is a pressing need for a uni- 
fying measurement framework in animal personality, and network models represent the current state-of-the-art.

\section{Conclusion}

I have reviewed measurement models in animal personality science, and demonstrated

how debates that personality is anthropomorphic, too descriptive, and lacking theoretical rationale can be linked to a lack of unified approach to measurement. Interestingly, these problems parallel similar debates in human personality, which are important to heed. Network models provide a particularly intriguing form of measurement model that may be of substantial interest to animal personality scientists. Not only does the network approach accommodate existing concepts such as behavioural repeatability and behavioural syndromes, it integrates with theoretical models such as state-dependent models and offers a more explicit hypothesis for the generation of behaviour than operational or latent variable approaches.

\section{References}

[1] David Sloan Wilson, Anne B Clark, Kristine Coleman, and Ted Dearstyne. Shyness and boldness in humans and other animals. Trends in Ecology 8 Evolution, 9(11):442-446, 1994.

[2] Ned A Dochtermann and Niels J Dingemanse. Behavioral syndromes as evolutionary constraints. Behavioral Ecology, 24(4):806-811, 2013.

[3] Sasha RX Dall, Alasdair I Houston, and John M McNamara. The behavioural ecology of personality: consistent individual differences from an adaptive perspective. Ecology letters, 7(8):734-739, 2004.

[4] Richard McElreath and Pontus Strimling. How noisy information and individual asymmetries can make 'personality' an adaptation: a simple model. Animal behaviour, 72(5):1135-1139, 2006.

[5] Niels J Dingemanse and Ned A Dochtermann. Individual behaviour: behavioural ecology meets quantitative genetics. In Anne Charmantier, Dany Garant, and Loeske EB Kruuk, editors, Quantitative genetics in the wild, pages 54-67. Oxford University Press Oxford, 2014.

[6] Andrew Sih, Alison M Bell, J Chadwick Johnson, and Robert E Ziemba. Behavioral syndromes: an integrative overview. The quarterly review of biology, 79(3):241-277, 2004. 
[7] Jana Uher. Comparative personality research: methodological approaches. European Journal of Personality, 22(5):427-455, 2008.

[8] Samuel D Gosling. From mice to men: what can we learn about personality from animal research? Psychological bulletin, 127(1):45, 2001.

[9] Sonja E Koski. How to measure animal personality and why does it matter? Integrating the psychological and biological approaches to animal personality. In From Genes to Animal Behavior, pages 115-136. Springer, 2011.

[10] Alecia J Carter, William E Feeney, Harry H Marshall, Guy Cowlishaw, and Robert Heinsohn. Animal personality: what are behavioural ecologists measuring? Biological Reviews, 88(2):465-475, 2013.

[11] Denis Réale, Simon M Reader, Daniel Sol, Peter T McDougall, and Niels J Dingemanse. Integrating animal temperament within ecology and evolution. Biological reviews, 82(2):291-318, 2007.

[12] Morgan David and Sasha RX Dall. Unravelling the philosophies underlying 'animal personality' studies: A brief re-appraisal of the field. Ethology, 122(1):19, 2016.

[13] Jana Uher. Individual behavioral phenotypes: an integrative meta-theoretical framework. Why behavioral syndromes are not analogs of personality. Developmental Psychobiology, 53(6):521-548, 2011.

[14] Madeleine Beekman and L Alex Jordan. Does the field of animal personality provide any new insights for behavioral ecology? Behavioral Ecology, 28(3):617623, 2017.

[15] David Crews. Animal Personalities: Behavior, Physiology, and Evolution. Claudio Carere and Dario Maestripieri, editors. Integrative and Comparative Biology, 53(5):873-875, 092013.

[16] Shinichi Nakagawa and Holger Schielzeth. Repeatability for Gaussian and nonGaussian data: a practical guide for biologists. Biological Reviews, 85(4):935956, 2010.

[17] Arne Jungwirth, Nina Wedell, Redouan Bshary, and Manfred Milinski. Animal personalities: an empty placeholder feigning understanding: a comment on Beekman and Jordan. Behavioral Ecology, 28(3):629-630, 2017.

[18] Dominique G Roche, Vincent Careau, and Sandra A Binning. Demystifying animal personality(or not): why individual variation matters to experimental biologists. Journal of Experimental Biology, 219(24):3832-3843, 2016. 
[19] Sasha RX Dall and Simon C Griffith. An empiricist guide to animal personality variation in ecology and evolution. Frontiers in Ecology and Evolution, 2:3, 2014.

[20] Alison M Bell. There is no special sauce: a comment on Beekman and Jordan. Behavioral Ecology, 28(3):626-627, 2017.

[21] Andrew Sih. Insights for behavioral ecology from behavioral syndromes: a comment on Beekman and Jordan. Behavioral Ecology, 28(3):627-628, 2017.

[22] Denny Borsboom. Measuring the mind: Conceptual issues in contemporary psychometrics. Cambridge University Press, 2005.

[23] Keith A Markus and Denny Borsboom. Frontiers of test validity theory: Measurement, causation, and meaning. Routledge, 2013.

[24] David J Hand. Statistics and the theory of measurement. Journal of the Royal Statistical Society: Series A (Statistics in Society), 159(3):445-473, 1996.

[25] Denis Réale, Niels J Dingemanse, Anahita JN Kazem, and Jonathan Wright. Evolutionary and ecological approaches to the study of personality. Philosophical Transactions of the Royal Society B: Biological Sciences, 2010.

[26] Niels J Dingemanse and Max Wolf. Recent models for adaptive personality differences: a review. Philosophical Transactions of the Royal Society B: Biological Sciences, 365(1560):3947-3958, 2010.

[27] Peter A Biro and Judy A Stamps. Do consistent individual differences in metabolic rate promote consistent individual differences in behavior? Trends in ecology \& evolution, 25(11):653-659, 2010.

[28] Judy Stamps and Ton GG Groothuis. The development of animal personality: relevance, concepts and perspectives. Biological Reviews, 85(2):301-325, 2010.

[29] Yimen G Araya-Ajoy and Niels J Dingemanse. Characterizing behavioural characters: an evolutionary framework. Proceedings of the Royal Society B: Biological Sciences, 281(1776):20132645, 2014.

[30] Liam R Dougherty and Lauren M Guillette. Linking personality and cognition: a meta-analysis. Philosophical Transactions of the Royal Society B: Biological Sciences, 373(1756):20170282, 2018.

[31] Fritz Trillmich, Thorben Müller, and Caroline Müller. Understanding the evolution of personality requires the study of mechanisms behind the development and life history of personality traits. Biology letters, 14(2):20170740, 2018. 
[32] Stephen John White, David John Pascall, and Alastair James Wilson. Towards a comparative approach to the structure of animal personality variation. Behavioral Ecology, 31(2):340-351, 2020.

[33] Kenneth S Kendler. Explanatory models for psychiatric illness. American Journal of Psychiatry, 165(6):695-702, 2008.

[34] Angélique OJ Cramer, Lourens J Waldorp, Han LJ Van Der Maas, and Denny Borsboom. Comorbidity: a network perspective. Behavioral and brain sciences, 33(2-3):137-150, 2010.

[35] Angélique OJ Cramer, Sophie Van der Sluis, Arjen Noordhof, Marieke Wichers, Nicole Geschwind, Steven H Aggen, Kenneth S Kendler, and Denny Borsboom. Dimensions of normal personality as networks in search of equilibrium: you can't like parties if you don't like people. European Journal of Personality, 26(4):414-431, 2012.

[36] Denny Borsboom, Angélique OJ Cramer, and Annemarie Kalis. Brain disorders? Not really: why network structures block reductionism in psychopathology research. Behavioral and Brain Sciences, 42, 2019.

[37] Conor Goold, Judit Vas, Christine Olsen, and Ruth C Newberry. Using network analysis to study behavioural phenotypes: an example using domestic dogs. Royal Society open science, 3(10):160268, 2016.

[38] Jordan S Martin, Jorg JM Massen, Vedrana Šlipogor, Thomas Bugnyar, Adrian V Jaeggi, and Sonja E Koski. The EGA+GNM framework: an integrative approach to modelling behavioural syndromes. Methods in Ecology and Evolution, 10(2):245-257, 2019.

[39] Niels J Dingemanse. The role of personality research in contemporary behavioral ecology: a comment on Beekman and Jordan. Behavioral Ecology, 28(3):624$625,2017$.

[40] David Sloan Wilson. Adaptive individual differences within single populations. Philosophical Transactions of the Royal Society of London. Series B: Biological Sciences, 353(1366):199-205, 1998.

[41] Niels J Dingemanse, Christiaan Both, Piet J Drent, and Joost M Tinbergen. Fitness consequences of avian personalities in a fluctuating environment. Proceedings of the Royal Society of London. Series B: Biological Sciences, 271(1541):847-852, 2004.

[42] John Paul Scott and John L Fuller. Genetics and the Social Behavior of the Dog. University of Chicago Press, 1965. 
[55] Niels J Dingemanse, Anahita JN Kazem, Denis Réale, and Jonathan Wright. Behavioural reaction norms: animal personality meets individual plasticity. Trends in ecology \&f evolution, 25(2):81-89, 2010. 
[56] Ian R Cleasby, Shinichi Nakagawa, and Holger Schielzeth. Quantifying the predictability of behaviour: statistical approaches for the study of betweenindividual variation in the within-individual variance. Methods in Ecology and Evolution, 6(1):27-37, 2015.

[57] Judy A Stamps, Mark Briffa, and Peter A Biro. Unpredictable animals: individual differences in intraindividual variability (iiv). Animal Behaviour, 83(6):1325-1334, 2012.

[58] David F Westneat, Jonathan Wright, and Niels J Dingemanse. The biology hidden inside residual within-individual phenotypic variation. Biological Reviews, 90(3):729-743, 2015.

[59] Niels J Dingemanse and Yimen G Araya-Ajoy. Interacting personalities: behavioural ecology meets quantitative genetics. Trends in ecology $\mathcal{E}$ evolution, 30(2):88-97, 2015.

[60] Yimen G Araya-Ajoy, Kimberley J Mathot, and Niels J Dingemanse. An approach to estimate short-term, long-term and reaction norm repeatability. Methods in Ecology and Evolution, 6(12):1462-1473, 2015.

[61] Hassen Allegue, Yimen G Araya-Ajoy, Niels J Dingemanse, Ned A Dochtermann, László Z Garamszegi, Shinichi Nakagawa, Denis Réale, Holger Schielzeth, and David F Westneat. Statistical quantification of individual differences (SQuID): an educational and statistical tool for understanding multilevel phenotypic data in linear mixed models. Methods in Ecology and Evolution, 8(2):257267, 2017.

[62] Alison M Bell, Shala J Hankison, and Kate L Laskowski. The repeatability of behaviour: a meta-analysis. Animal behaviour, 77(4):771-783, 2009.

[63] Peter A Biro and Judy A Stamps. Using repeatability to study physiological and behavioural traits: ignore time-related change at your peril. Animal Behaviour, 105:223-230, 2015.

[64] Percy Williams Bridgman. The Logic of Modern Physics, volume 3. Macmillan New York, 1927.

[65] Tim B Rogers. Operationism in psychology: a discussion of contextual antecedents and an historical interpretation of its longevity. Journal of the History of the Behavioral Sciences, 25(2):139-153, 1989.

[66] Stanley S Stevens. The operational basis of psychology. The American Journal of Psychology, 47(2):323-330, 1935.

[67] LJ Russell. The logic of modern physics., 1928. 
[68] H Israel and B Goldstein. Operationism in psychology. Psychological Review, $51(3): 177,1944$.

[69] Christopher D Green. Of immortal mythological beasts: operationism in psychology. Theory \& Psychology, 2(3):291-320, 1992.

[70] Joel Michell. Quantitative science and the definition of measurement in psychology. British journal of Psychology, 88(3):355-383, 1997.

[71] Christopher D Green. Operationism again: what did Bridgman say? What did Bridgman need? Theory \& Psychology, 11(1):45-51, 2001.

[72] Andrew Maul, David Torres Irribarra, and Mark Wilson. On the philosophical foundations of psychological measurement. Measurement, 79:311-320, 2016.

[73] Mark H Bickhard. The tragedy of operationalism. Theory E Psychology, 11(1):35-44, 2001.

[74] Denny Borsboom. The attack of the psychometricians. Psychometrika, $71(3): 425,2006$.

[75] FR Stafleu, FJ Grommers, and J Vorstenbosch. Animal welfare: evolution and erosion of a moral concept. Animal Welfare, 5:225-234, 1996.

[76] Denny Borsboom, Angélique OJ Cramer, Rogier A Kievit, Annemarie Zand Scholten, and Sanja Franić. The end of construct validity. In The Concept of Validity: Revisions, New Directions and Applications, Oct, 2008. IAP Information Age Publishing, 2009.

[77] Ned A Dochtermann and Andrew B Nelson. Multiple facets of exploratory behavior in house crickets (A cheta domesticus): split personalities or simply different behaviors? Ethology, 120(11):1110-1117, 2014.

[78] Sergey V Budaev. Using principal components and factor analysis in animal behaviour research: caveats and guidelines. Ethology, 116(5):472-480, 2010.

[79] Kenneth Bollen and Richard Lennox. Conventional wisdom on measurement: a structural equation perspective. Psychological bulletin, 110(2):305, 1991.

[80] Charles Spearman. "General intelligence" objectively determined and measured. The American Journal of Psychology, 15:201-293, 1904.

[81] Charles Spearman. The abilities of man, volume 6. Macmillan New York, 1927.

[82] Robert R McCrae and Paul T Costa. Personality trait structure as a human universal. American psychologist, 52(5):509, 1997. 
[83] Ned A Dochtermann and Stephen H Jenkins. Behavioural syndromes in Merriam's kangaroo rats (Dipodomys merriami): a test of competing hypotheses. Proceedings of the Royal Society B: Biological Sciences, 274(1623):2343-2349, 2007.

[84] Niels J Dingemanse, Ned Dochtermann, and Jonathan Wright. A method for exploring the structure of behavioural syndromes to allow formal comparison within and between data sets. Animal Behaviour, 79(2):439-450, 2010.

[85] Yimen G Araya-Ajoy, Peter Sjolte Ranke, Thomas Kvalnes, Bernt Rønning, Håkon Holand, Ane Marlene Myhre, Henrik Pärn, Henrik Jensen, Thor Harald Ringsby, Bernt-Erik Sæther, et al. Characterizing morphological (co) variation using structural equation models: body size, allometric relationships and evolvability in a house sparrow metapopulation. Evolution, 73(3):452-466, 2019.

[86] Simon Boag. Explanation in personality psychology: 'verbal magic' and the five-factor model. Philosophical Psychology, 24(2):223-243, 2011.

[87] Richard P Bagozzi and Jeffrey R Edwards. A general approach for representing constructs in organizational research. Organizational research methods, 1(1):4587, 1998.

[88] Kristopher J Preacher and Robert C MacCallum. Repairing Tom Swift's electric factor analysis machine. Understanding statistics: Statistical issues in psychology, education, and the social sciences, 2(1):13-43, 2003.

[89] Keith F Widaman. Common factor analysis versus principal component analysis: differential bias in representing model parameters? Multivariate behavioral research, 28(3):263-311, 1993.

[90] F Blake Morton and Drew Altschul. Data reduction analyses of animal behaviour: avoiding Kaiser's criterion and adopting more robust automated methods. Animal behaviour, 149:89-95, 2019.

[91] Mats Björklund. Be careful with your principal components. Evolution, 73(10):2151-2158, 2019.

[92] Wayne F Velicer and Douglas N Jackson. Component analysis versus common factor analysis: some further observations. Multivariate Behavioral Research, 25(1):97-114, 1990.

[93] Richard L Gorsuch. Common factor analysis versus component analysis: some well and little known facts. Multivariate Behavioral Research, 25(1):33-39, 1990.

[94] Denny Borsboom, Gideon J Mellenbergh, and Jaap Van Heerden. The concept of validity. Psychological review, 111(4):1061, 2004. 
[95] Leandre R Fabrigar, Duane T Wegener, Robert C MacCallum, and Erin J Strahan. Evaluating the use of exploratory factor analysis in psychological research. Psychological methods, 4(3):272, 1999.

[96] David J Bartholomew. Factor analysis for categorical data. Journal of the Royal Statistical Society: Series B (Methodological), 42(3):293-312, 1980.

[97] William Meredith. Measurement invariance, factor analysis and factorial invariance. Psychometrika, 58(4):525-543, 1993.

[98] Steven P Reise, Keith F Widaman, and Robin H Pugh. Confirmatory factor analysis and item response theory: two approaches for exploring measurement invariance. Psychological bulletin, 114(3):552, 1993.

[99] Fritz Drasgow. Study of the measurement bias of two standardized psychological tests. Journal of Applied psychology, 72(1):19, 1987.

[100] Jelte M Wicherts. The importance of measurement invariance in neurocognitive ability testing. The Clinical Neuropsychologist, 30(7):1006-1016, 2016.

[101] Jelte M Wicherts and Conor V Dolan. Measurement invariance in confirmatory factor analysis: an illustration using IQ test performance of minorities. Educational Measurement: Issues and Practice, 29(3):39-47, 2010.

[102] Conor Goold and Ruth C Newberry. Aggressiveness as a latent personality trait of domestic dogs: testing local independence and measurement invariance. PloS one, 12(8), 2017.

[103] Peter CM Molenaar. A dynamic factor model for the analysis of multivariate time series. Psychometrika, 50(2):181-202, 1985.

[104] Janne Adolf, Noémi K Schuurman, Peter Borkenau, Denny Borsboom, and Conor V Dolan. Measurement invariance within and between individuals: a distinct problem in testing the equivalence of intra-and inter-individual model structures. Frontiers in psychology, 5:883, 2014.

[105] Peter CM Molenaar. A manifesto on psychology as idiographic science: bringing the person back into scientific psychology, this time forever. Measurement, 2(4):201-218, 2004.

[106] WS Robinson. Ecological correlations and individual behavior. American Sociological Review, 15(195):351-57, 1950.

[107] Hanan C Selvin. Durkheim's suicide and problems of empirical research. American journal of sociology, 63(6):607-619, 1958. 
[108] Cristiano Mauro Assis Gomes, Jhonys de Araujo, Elizabeth do Nascimento, and Enio Galinkin Jelihovschi. Routine psychological testing of the individual is not valid. Psychological reports, 122(4):1576-1593, 2019.

[109] Godfrey H Thomson. A hierarchy without a general factor. British Journal of Psychology, 8(3):271, 1916.

[110] Han LJ Van Der Maas, Conor V Dolan, Raoul PPP Grasman, Jelte M Wicherts, Hilde M Huizenga, and Maartje EJ Raijmakers. A dynamical model of general intelligence: the positive manifold of intelligence by mutualism. Psychological review, 113(4):842, 2006.

[111] Rogier A Kievit, Hilko Van Rooijen, Jelte M Wicherts, Lourens J Waldorp, Kees-Jan Kan, H Steven Scholte, and Denny Borsboom. Intelligence and the brain: a model-based approach. Cognitive Neuroscience, 3(2):89-97, 2012.

[112] Chandra Sripada, Mike Angstadt, Saige Rutherford, Daniel Kessler, Yura Kim, Mike Yee, and Elizaveta Levina. Basic units of inter-individual variation in resting state connectomes. Scientific reports, 9(1):1-12, 2019.

[113] Theodore D Satterthwaite, Cedric H Xia, and Danielle S Bassett. Personalized neuroscience: common and individual-specific features in functional brain networks. Neuron, 98(2):243-245, 2018.

[114] Julien Dubois, Paola Galdi, Lynn K Paul, and Ralph Adolphs. A distributed brain network predicts general intelligence from resting-state human neuroimaging data. Philosophical Transactions of the Royal Society B: Biological Sciences, 373(1756):20170284, 2018.

[115] Aikaterini Fotopoulou. Time to get rid of the 'modular' in neuropsychology: a unified theory of anosognosia as aberrant predictive coding. Journal of neuropsychology, 8(1):1-19, 2014.

[116] J Benjamin Hutchinson and Lisa Feldman Barrett. The power of predictions: an emerging paradigm for psychological research. Current directions in psychological science, 28(3):280-291, 2019.

[117] Gerald M Edelman and Joseph A Gally. Degeneracy and complexity in biological systems. Proceedings of the National Academy of Sciences, 98(24):1376313768, 2001.

[118] Karl J Friston and Cathy J Price. Degeneracy and redundancy in cognitive anatomy. Trends in cognitive sciences, 4(7):151-152, 2003.

[119] Susan Nolen-Hoeksema and Edward R Watkins. A heuristic for developing transdiagnostic models of psychopathology: explaining multifinality and divergent trajectories. Perspectives on Psychological Science, 6(6):589-609, 2011. 
[120] Alfred North Whitehead. Process and Reality. Macmillan, 1929.

[121] Mark Flynn. The concept of intelligence in psychology as a fallacy of misplaced concreteness. Interchange, 28(2-3):231-244, 1997.

[122] David B Clark and James R Kellner. Tropical forest biomass estimation and the fallacy of misplaced concreteness. Journal of Vegetation Science, 23(6):11911196, 2012.

[123] Fritz Machlup. Equilibrium and disequilibrium: misplaced concreteness and disguised politics. The Economic Journal, 68(269):1-24, 1958.

[124] Michael T Turvey and Peter N Kugler. Chapter IV: an ecological approach to perception and action. In Advances in Psychology, volume 17, pages 373-412. Elsevier, 1984.

[125] Paul E Smaldino. Models are stupid, and we need more of them. Computational social psychology, pages 311-331, 2017.

[126] Paul Smaldino. Better methods can't make up for mediocre theory. Nature, $575(7781): 9,2019$.

[127] Sabrina Golonka and Andrew D Wilson. Ecological representations. Ecological Psychology, 31(3):235-253, 2019.

[128] Max Wolf, G Sander Van Doorn, Olof Leimar, and Franz J Weissing. Life-history trade-offs favour the evolution of animal personalities. Nature, 447(7144):581-584, 2007.

[129] Max Wolf and Franz J Weissing. An explanatory framework for adaptive personality differences. Philosophical Transactions of the Royal Society B: Biological Sciences, 365(1560):3959-3968, 2010.

[130] Andrew Sih, Kimberley J Mathot, María Moirón, Pierre-Olivier Montiglio, Max Wolf, and Niels J Dingemanse. Animal personality and state-behaviour feedbacks: a review and guide for empiricists. Trends in ecology $\&$ evolution, 30(1):50-60, 2015.

[131] Alan A Cohen, Lynn B Martin, John C Wingfield, Scott R McWilliams, and Jennifer A Dunne. Physiological regulatory networks: ecological roles and evolutionary constraints. Trends in ecology \& evolution, 27(8):428-435, 2012.

[132] Albert-László Barabási. The network takeover. Nature Physics, 8(1):14-16, 2012. 
[133] Amir Bashan, Ronny P Bartsch, Jan W Kantelhardt, Shlomo Havlin, and Plamen Ch Ivanov. Network physiology reveals relations between network topology and physiological function. Nature communications, 3(1):1-9, 2012.

945

[134] Fritjof Capra and Pier Luigi Luisi. The Systems View of Life: A Unifying Vision. Cambridge University Press, 2014.

[135] Stuart A Kauffman. The Origins of Order: Self-organization and Selection in Evolution. Oxford University Press, USA, 1993.

[136] Tobias Uller, Armin P Moczek, Richard A Watson, Paul M Brakefield, and Kevin N Laland. Developmental bias and evolution: a regulatory network perspective. Genetics, 209(4):949-966, 2018.

[137] Nicholas Rescher. Process Metaphysics: An Introduction to Process Philosophy. Suny Press, 1996.

[138] John Dupré. Processes of Life: Essays in the Philosophy of Biology. Oxford University Press, 2012.

[139] John Dupré. The role of behaviour in the recurrence of biological processes. Biological Journal of the Linnean Society, 112(2):306-314, 2014.

[140] Eric Bapteste and John Dupré. Towards a processual microbial ontology. Biology $\mathscr{E}$ Philosophy, 28(2):379-404, 2013.

[141] Judea Pearl. Causality: Models, Reasoning, and Inference. Cambridge University Press, 2000.

[142] Angélique OJ Cramer, Claudia D Van Borkulo, Erik J Giltay, Han LJ Van Der Maas, Kenneth S Kendler, Marten Scheffer, and Denny Borsboom. Major depression as a complex dynamic system. PloS one, 11(12):e0167490, 2016.

[143] Eiko I Fried, Claudia D van Borkulo, Angélique OJ Cramer, Lynn Boschloo, Robert A Schoevers, and Denny Borsboom. Mental disorders as networks of problems: a review of recent insights. Social Psychiatry and Psychiatric Epidemiology, 52(1):1-10, 2017.

[144] Robin N Groen, Oisín Ryan, Johanna TW Wigman, Harriëtte Riese, Brenda WJH Penninx, Erik J Giltay, Marieke Wichers, and Catharina A Hartman. Comorbidity between depression and anxiety: assessing the role of bridge mental states in dynamic psychological networks. BMC medicine, 18(1):1-17, 2020. 
[145] Verena D Schmittmann, Angélique OJ Cramer, Lourens J Waldorp, Sacha Epskamp, Rogier A Kievit, and Denny Borsboom. Deconstructing the construct: a network perspective on psychological phenomena. New ideas in psychology, 31(1):43-53, 2013.

[146] Denny Borsboom and Angélique OJ Cramer. Network analysis: an integrative approach to the structure of psychopathology. Annual review of clinical psychology, 9:91-121, 2013.

[147] M Marsman, D Borsboom, J Kruis, S Epskamp, R van Bork, LJ Waldorp, HLJ van der Maas, and G Maris. An introduction to network psychometrics: relating ising network models to item response theory models. Multivariate behavioral research, 53(1):15-35, 2018.

[148] Laura F Bringmann and Markus I Eronen. Dont blame the model: reconsidering the network approach to psychopathology. Psychological Review, 125(4):606, 2018.

[149] Richard Boyd. Realism, anti-foundationalism and the enthusiasm for natural kinds. Philosophical studies, 61(1-2):127-148, 1991.

[150] Kenneth S Kendler, Peter Zachar, and C Craver. What kinds of things are psychiatric disorders? Toward a Philosophical Approach to Psychiatry: The Writings of Kenneth Kendler, page 62, 2011.

[151] Sacha Epskamp, Mijke Rhemtulla, and Denny Borsboom. Generalized network psychometrics: combining network and latent variable models. Psychometrika, 82(4):904-927, 2017.

[152] Han LJ Van Der Maas, Kees-Jan Kan, Maarten Marsman, and Claire E Stevenson. Network models for cognitive development and intelligence. Journal of Intelligence, 5(2):16, 2017.

[153] Stefan G Hofmann, Joshua Curtiss, and Richard J McNally. A complex network perspective on clinical science. Perspectives on Psychological Science, 11(5):597$605,2016$.

[154] Laura F Bringmann, Nathalie Vissers, Marieke Wichers, Nicole Geschwind, Peter Kuppens, Frenk Peeters, Denny Borsboom, and Francis Tuerlinckx. A network approach to psychopathology: new insights into clinical longitudinal data. PloS one, 8(4):e60188, 2013.

[155] Adela-Maria Isvoranu, Denny Borsboom, Jim van Os, and Sinan Guloksuz. A network approach to environmental impact in psychotic disorder: brief theoretical framework. Schizophrenia Bulletin, 42(4):870-873, 2016. 
[156] Miriam K Forbes, Aidan GC Wright, Kristian E Markon, and Robert F Krueger. Evidence that psychopathology symptom networks have limited replicability. Journal of Abnormal Psychology, 126(7):969, 2017.

[157] Denny Borsboom, Eiko I Fried, Sacha Epskamp, Lourens J Waldorp, Claudia D van Borkulo, Han LJ van der Maas, and Angélique OJ Cramer. False alarm? A comprehensive reanalysis of "Evidence that psychopathology symptom networks have limited replicability" by Forbes, Wright, Markon, and Krueger (2017). Journal of Abnormal Psychology, 126(7):989-999, 2017.

[158] Matthew R Wilkins, Daizaburo Shizuka, Maxwell B Joseph, Joanna K Hubbard, and Rebecca J Safran. Multimodal signalling in the north american barn swallow: a phenotype network approach. Proceedings of the Royal Society B: Biological Sciences, 282(1816):20151574, 2015.

[159] Eileen A Hebets, Andrew B Barron, Christopher N Balakrishnan, Mark E Hauber, Paul H Mason, and Kim L Hoke. A systems approach to animal communication. Proceedings of the Royal Society B: Biological Sciences, 283(1826):20152889, 2016. 